\title{
Structure-function analysis of DNA helicase HELQ: A new diagnostic marker in ovarian cancer
}

\author{
YA-PING LI ${ }^{1,2}$, JUN-JUAN YANG ${ }^{3}$, HUI XU ${ }^{4}$, EN-YU GUO ${ }^{5}$ and YAN YU ${ }^{1}$ \\ ${ }^{1}$ School of Public Health, Health Science Center of Xi'an Jiaotong University, Xi'an, Shaanxi 710061; \\ ${ }^{2}$ Department of Obstetrics and Gynecology, Xi'an Central Hospital, Xi'an, Shaanxi 710003; \\ ${ }^{3}$ Department of Obstetrics and Gynecology, Women \& Infants Hospital of Zhengzhou, Zhengzhou, Henan 450053; \\ Departments of ${ }^{4}$ Obstetrics and Gynecology and ${ }^{5}$ Equipment, Zhengzhou People's Hospital, \\ Zhengzhou, Henan 450003, P.R. China
}

Received April 21,2015; Accepted September 9, 2016

DOI: $10.3892 / 01.2016 .5224$

\begin{abstract}
It has been previously reported that a deficiency of the helicase, POLQ-like (HELQ) gene increases the risk of ovarian cancer. The present study aimed to explore the structure-function association of HELQ and discuss the effect of molecular structure on the occurrence of tumors. ExPASy tools were employed to analyze the physicochemical properties and secondary structure of the genes. PHYRE2 Protein Fold Recognition Server was used to construct the three-dimensional model and find the ligand-binding sites of HELQ. In addition, the potential functions corresponding to these structures were excavated by comparing and analyzing protein domains. The HELQ protein is located in the cytoplasm (56.5\%) and nucleus (21.7\%). HELQ has 4 conserved domains, consisting of DEXDc, HELICc, HHH_5 and PRK02362, which contain the adenosine triphosphate (ATP) binding site, nucleotide binding region and putative $\mathrm{Mg}^{2+}$ binding site. In the secondary structure, it was found that HELQ was mainly composed of $\alpha$ helix (46.68\%) and random coils (43.05\%), with only $10.26 \%$ extended strand. According to 3DLigandSite Server, the ligand binding sites appeared in ILE333, LYS335, TYR337, SER362, LEU367, LYS397, GLN340, GLY363, GLY364 and ASN678 of the amino acid sequence. Among the functional protein association networks, regulator of telomere elongation helicase 1 , family with sequence similarity 175 member A, small ubiquitin-like modifier 1, DNA polymerase $v$ and coiled-coil domain containing 158 were involved and co-expressed with HELQ. PredictProtein analysis indicated that the dominant
\end{abstract}

Correspondence to: Dr Yan Yu, School of Public Health, Health Science Center of Xi'an Jiaotong University, 76 Yanta Road, Xi'an, Shaanxi 710061, P.R. China

E-mail: guoeny01@163.com

Key words: structure-function, DNA helicase, HELQ, ovarian cancer, bioinformatics functions of HELQ were ATP-dependent helicase activity and participation in the DNA repair process. Characteristics of the HELQ protein were obtained by bioinformatics analysis, based on which the role of HELQ in DNA replication, DNA repair and maintenance of genomic stability was explored. It was concluded that modulation the function of HELQ helicase may be used in the treatment of ovarian cancer.

\section{Introduction}

Ovarian cancer is the second most common gynecological cancer worldwide, accounting for $\sim 3 \%$ of all female cancer cases, with the typical age of diagnosis being 63-years-old. It is challenging to diagnose ovarian cancer at an early stage, and this cancer has a poor prognosis, with a five-year survival rate of $\sim 47 \%$ (1). In order to provide a foundation for detecting and treating ovarian cancer, studies investigating the etiology of the disease are required. Previously, a study by the London Research Institute (Cancer Research UK, London, UK) found helicase, POLQ-like (HELQ) to be a novel gene that prevents ovarian cancer (2). In this study, the possibility of ovarian cancer in mice was increased two-fold when a copy of the HELQ gene was missing. Even the deficiency of one copy could lead to the formation of an increased number of tumors in mice. According to this study, detection of HELQ gene deficiency may be adopted to screen for ovarian cancer patients among women in the future, if HELQ helicase performs the same role in humans and mice (2).

HELQ is a superfamily II DNA helicase that was first identified in the human and mouse genomes through its homology to mutagen-sensitive 308 (Mus308) (3), a DNA repair enzyme required for DNA interstrand crosslink (ICL) resistance in Drosophila melanogaster (4,5). DNA ICLs are particularly toxic as they disrupt genetic information on strands, potently inhibiting DNA replication and transcription. In normal cells, DNA ICLs and DNA damage often lead to the occurrence of cancer (6). However, DNA repair is an important reaction following DNA damage, which may cause the damaged DNA to revert to the original appearance and perform the original function (7). DNA helicases play an important role in this 
process. HELQ, as a DNA helicase, has been studied from the perspective of association with cancer. Previous genome-wide association studies have identified single nucleotide polymorphisms at loci within or near the HELQ gene that are associated with an increased risk of several different cancers, including upper aerodigestive tract cancers and head and neck cancers (8-11).

To assess the association between the structure of HELQ and the carcinogenesis of ovarian cancer, bioinformatics methods were used to analyze this association from a theoretical angle in the present study. It was expected that such a study may provide direction and basis for the clinical treatment of ovarian cancer.

\section{Materials and methods}

Gene sequence. The HELQ gene sequence was obtained from the Nucleotide resource of the NationalCenter for Biotechnology Information (NCBI; http://www.ncbi.nlm.nih.gov/nucleotide), using the GenBank accession number AF436845.1 and Gene ID 113510. The HELQ gene is also termed HEL308.

Bioinformatics analysis. The BioEdit software (http://www.mbio.ncsu.edu/bioedit/bioedit.html) was used for the analysis of open reading frames. The ExPASy tools ProtParam (http://web.expasy.org/protparam/) and ProtScale (http://web.expasy.org/protscale/) were used to compute gene features, including the amino acid composition, molecular mass, isoelectric point, hydrophobicity/hydrophilicity, instability index and aliphatic index. The SignalP-HMM Server was used to predict the signal peptides (12). The transmembrane region was analyzed using the TMHMM Server V.2.0 system (http://www.cbs.dtu.dk/services/TMHMM/). Subcellular localization was predicted using the Protein Subcellular Localization Prediction Tool (PSORT) database (http://www.psort.org/).TheSimpleModularArchitectureResearch Tool (SMART; http://smart.embl-heidelberg.de/) was used for analysis of protein domains. Hopfield Neural Network (HNN) was used to make the secondary structure predictions (13). PHYRE2 Protein Fold Recognition Server online software (http://www.sbg.bio.ic.ac.uk/phyre2/html/page.cgi?id=index) was employed to obtain the three-dimensional structure of HELQ and the 3DLigandSite Server (http://www.sbg.bio. ic.ac.uk/3dligandsite/) was used to construct the three-dimensional ligand binding model. STRING9.0 interactive database (http://string-db.org/) was utilized to find the associated proteins. Gene ontology analysis was performed using PredictProtein software (https://www.predictprotein.org/).

\section{Results}

Analysis of physicochemical properties of HELQ. Through NCBI database retrieval, the whole nucleotide sequence and amino acid sequence of HELQ were obtained, with a total sequence of 3,591 bp encoding 1,101 amino acid residues. Open reading frame (ORF) prediction and BioEdit analysis showed that the HELQ gene contained 15 ORFs. ProtParam predicted that the molecular weight of the HELQ gene was $124,175.3 \mathrm{Da}$, the theoretical isoelectric point was 6.12 , the content of leucine (Leu) was $16.2 \%$ of the total components, acidic amino acids were more common than basic amino acids, and the instability index was 45.55. Therefore, it was inferred that HELQ was an unstable and acidic protein. In addition, the value of grand average of hydropathicity was -0.317 and the aliphatic index was 92.34 , which indicated that HELQ is liposoluble.

Subcellular localization and motif prediction. The location of proteins in cells is closely associated with the function of proteins. Subcellular localization analysis of PSORT prediction showed that HELQ was mainly distributed in the nucleus, mitochondrial matrix space, microbody (peroxisome) and mitochondrial inner membrane (Table I). HELQ was located in areas with the presence of DNA.

As for motifs, the functional structure domain database of ScanProsite predicted that the HELQ protein contained two functional domains, consisting of Helicase_ATP-Bind_1 and Helicase_Cter, and these domains were responsible for binding and hydrolyzing ATP, respectively, which conformed to the characteristic of DNA helicase depending on ATP hydrolysis (Fig. 1A). Domain architecture analysis using SMART showed that 5 main domains could be found in the HELQ sequence, with other features not shown in the diagram (Fig. 1B) due to overlap. By searching the NCBI Conserved Domains Database, 4 domains were found, which were DEXDc, HELICc, HHH-5 and PRK02362 (Fig. 1C). According to the prediction of SignalP-HMM and TMHMM, the HELQ protein has no evident signal peptide and transmembrane domain.

Structural analysis of the HELQ protein. HNN was used to analyze the secondary structure of the HELQ protein. Results showed that HELQ was mainly composed of $\alpha$-helixes $(46.48 \%)$ and random coils (43.05\%), with extended strands accounting for only $10.26 \%$ of the structure (Fig. 2). According to the prediction of the tertiary structure using the threading method in the PHYRE2 Protein Fold Recognition Server, the best template protein was c2va8A (Protein Data Bank code), which had high homology with HELQ (Fig. 3). The 3DLigandSite Server was used to construct the three-dimensional ligand binding model (Fig. 4). It was found that the ligand binding sites were distributed over ILE333, LYS335, TYR337, GLN340, SER362, GLY363, GLY364, LYS365, THR366, LEU367, LYS397 and ASN678. Heterogens present in the predicted binding sites consisted of 10 adenosine diphosphate (ADP), $14 \mathrm{Mg}^{2+}(\mathrm{MG})$ and 3 adenosine monophosphate (AMP). The functional domains Helicase_ATP-Bind_1, Helicase_Cter and HHH_5 were separated from the HELQ sequence and were respectively analyzed by building ligand binding models. The results suggested that ligand binding sites in the Helicase ATP-Bind_1 domain distributed over SER17, GLY18, GLY19, LYS20, THR21, LEU22, LYS52 and the heterogens present in the predicted binding sites contained 12 ADP, 14 MG and 3 AMP. The binding site present in the Helicase_Cter domain was ASN113, and the heterogens contained 4 ADP, $12 \mathrm{MG}$ and 1 ATP. The binding site in the HHH_5 domain was GLU15 and the heterogens contained $7 \mathrm{MG}$ and $33 \mathrm{Ca}^{2+}$. The activity of HELQ is dependent on the binding of ATP by the Helicase_Cter domain to supply energy. When functional domains of HELQ are not complete, DNA replication cannot progress normally. 


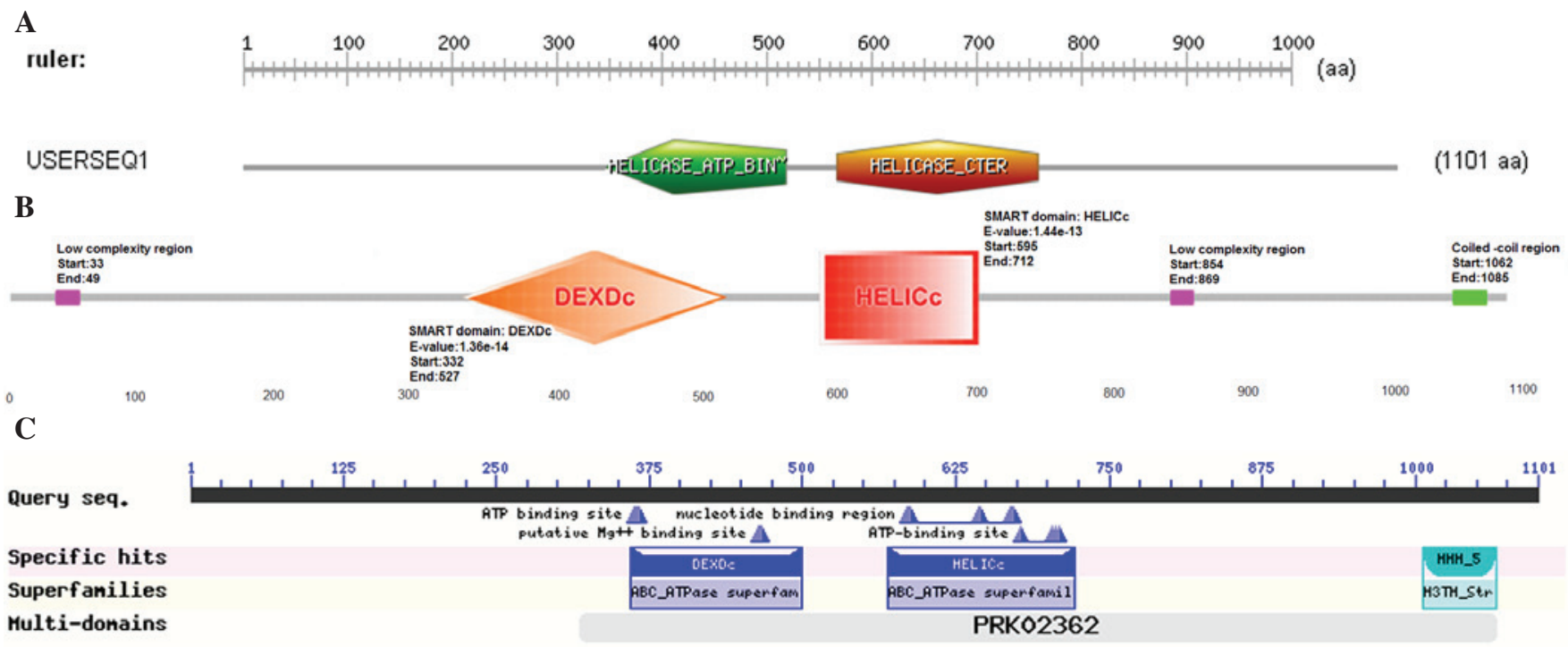

Figure 1. Prediction of functional domains. (A) ScanProsite; (B) Simple Modular Architecture Research Tool; (C) National Center for Biotechnology Information conserved domains.

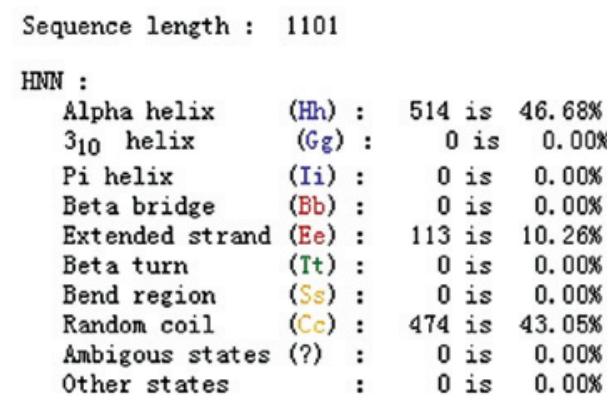

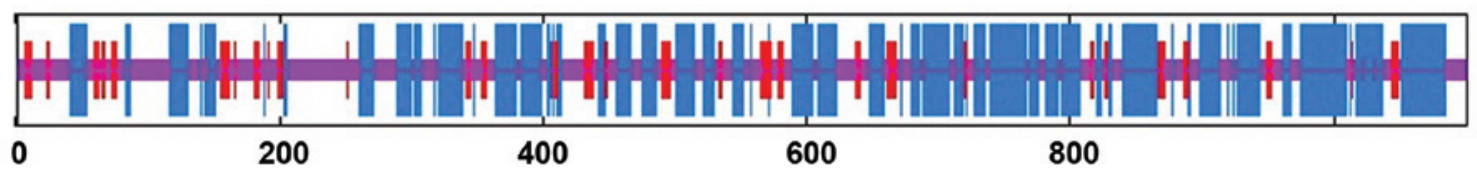

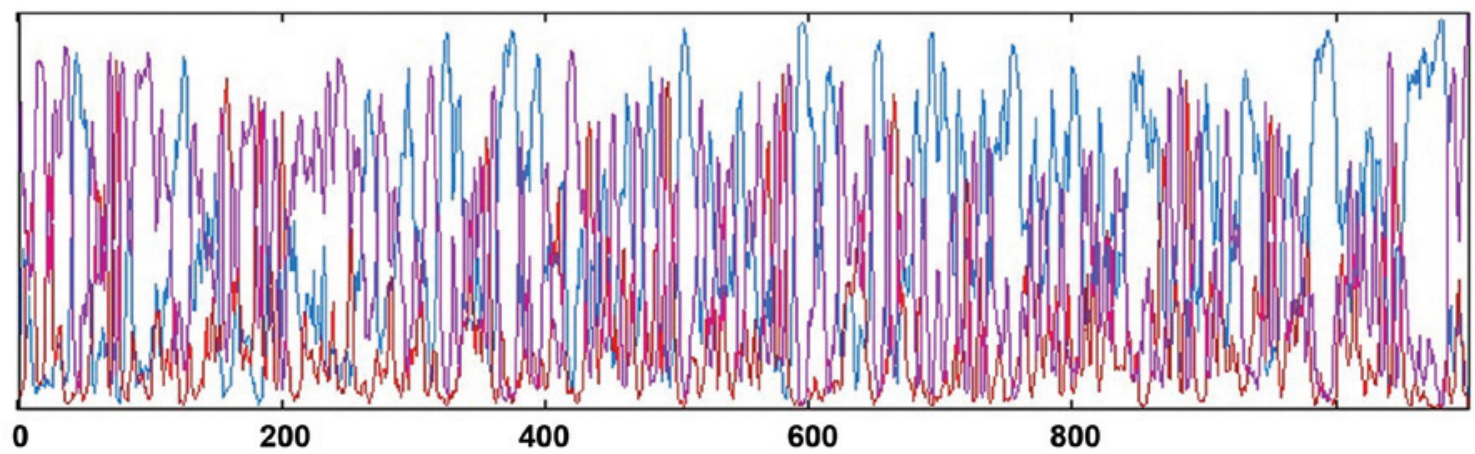

Figure 2. Secondary structure of the helicase, POLQ-like protein.

Prediction of protein-protein interactions. STRING9.0 interactive database was utilized to determine the functional protein association networks. Proteins that interact with HELQ mainly included regulator of telomere elongation helicase 1 (RTEL1), family with sequence similarity 175 member A (FAM175A), small ubiquitin-like modifier 1 (SUMO1), DNA polymerase $v$ (POLN) and coiled-coil domain containing 158 (Fig. 5). They were coexpressed and had similar functions in repairing the DNA lesions appearing in the process of DNA replication during cell proliferation.

Gene ontology analysis. Gene ontology analysis was performed using PredictProtein software. Molecular function ontology showed that the activity of HELQ mainly consisted of 
TableI.Subcellularlocalization ofhelicase,POLQ-likeencoding product.

\begin{tabular}{lc}
\hline Subcellular localization & Certainty \\
\hline Nucleus & 0.600 \\
Mitochondrial matrix space & 0.510 \\
Microbody (peroxisome) & 0.300 \\
Mitochondrial inner membrane & 0.234 \\
\hline
\end{tabular}

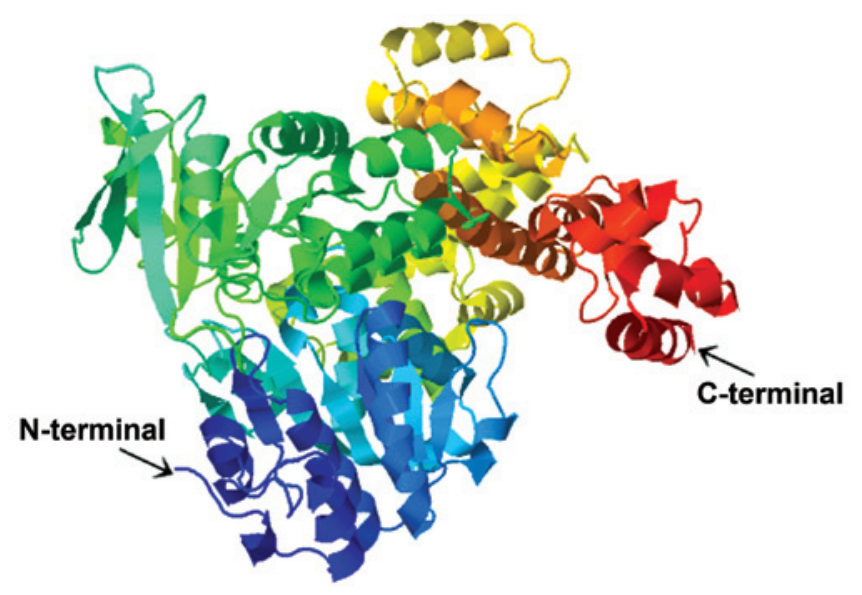

Figure 3. Tertiary structure of the helicase, POLQ-like protein.
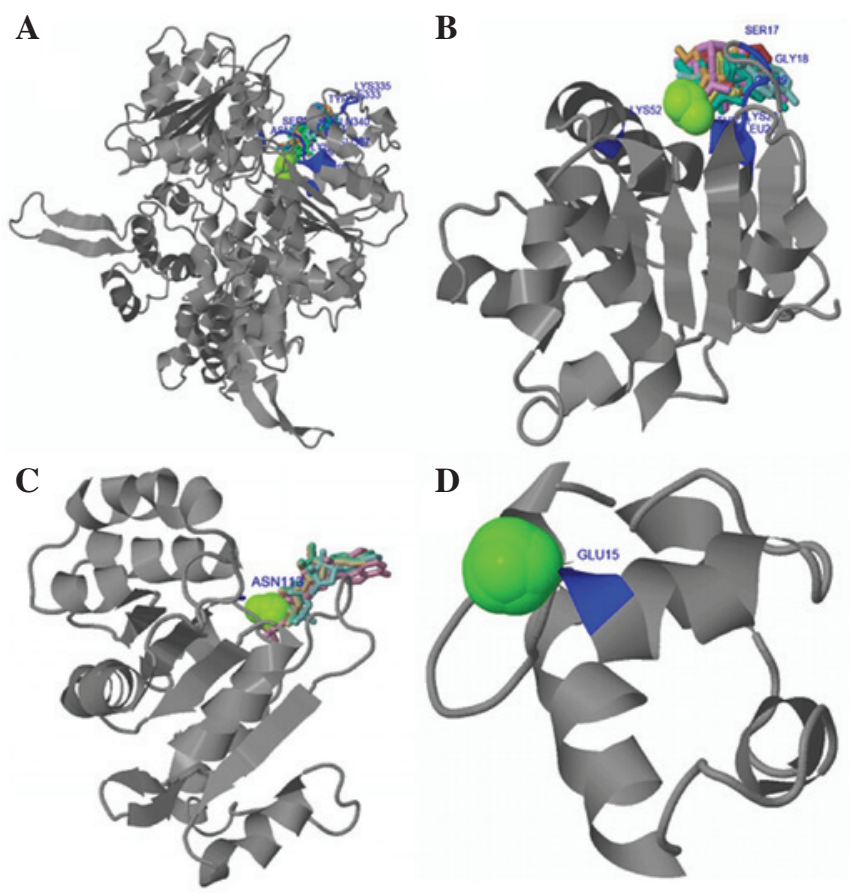

Figure 4. Construction of three-dimensional ligand binding models of (A) helicase, POLQ-like, (B) Helicase_ATP-Bind_1 domain, (C) Helicase_Cter domain and (D) $\mathrm{HHH}_{-} 5$ domain.

protein binding, and helicase, ATP-dependent RNA helicase, DNA strand annealing, ATPase, ATP-dependent helicase, RNA helicase and RNA-dependent ATPase activity (Table II). In addition, biological process ontology analysis indicated
Table II. Molecular function ontology.

\begin{tabular}{|c|c|c|}
\hline GO ID & GO term & Reliability, \% \\
\hline GO:0005515 & Protein binding & 26 \\
\hline GO:0004386 & Helicase activity & 17 \\
\hline GO:0004004 & $\begin{array}{l}\text { ATP-dependent RNA } \\
\text { helicase activity }\end{array}$ & 14 \\
\hline GO:0000739 & $\begin{array}{l}\text { DNA strand annealing } \\
\text { activity }\end{array}$ & 14 \\
\hline GO:0016887 & ATPase activity & 14 \\
\hline GO:0008026 & $\begin{array}{l}\text { ATP-dependent helicase } \\
\text { activity }\end{array}$ & 12 \\
\hline GO:0003724 & RNA helicase activity & 11 \\
\hline GO:0008186 & $\begin{array}{l}\text { RNA-dependent ATPase } \\
\text { activity }\end{array}$ & 10 \\
\hline GO:0042802 & identical protein binding & 6 \\
\hline GO:0043140 & $\begin{array}{l}\text { ATP-dependent 3'-5' DNA } \\
\text { helicase activity }\end{array}$ & 4 \\
\hline GO:0043621 & Protein self-association & 4 \\
\hline GO:0005524 & ATP binding & 4 \\
\hline GO:0043008 & $\begin{array}{l}\text { ATP-dependent protein } \\
\text { binding }\end{array}$ & 4 \\
\hline GO:0008432 & JUN kinase binding & 3 \\
\hline GO:0017151 & $\begin{array}{l}\text { DEAD/H-box RNA } \\
\text { helicase binding }\end{array}$ & 3 \\
\hline GO:0003678 & DNA helicase activity & 3 \\
\hline GO:0030621 & U4 snRNA binding & 2 \\
\hline GO:0003712 & $\begin{array}{l}\text { Transcription cofactor } \\
\text { activity }\end{array}$ & 2 \\
\hline GO:0017070 & U6 snRNA binding & 2 \\
\hline GO:0003743 & $\begin{array}{l}\text { Translation initiation factor } \\
\text { activity }\end{array}$ & 2 \\
\hline GO:0003730 & mRNA 3'-UTR binding & 2 \\
\hline GO:0003677 & DNA binding & 2 \\
\hline GO:0000339 & RNA cap binding & 2 \\
\hline GO:0003723 & RNA binding & 2 \\
\hline GO:0008143 & Poly(A) RNA binding & 2 \\
\hline GO:0008094 & $\begin{array}{l}\text { DNA-dependent ATPase } \\
\text { activity }\end{array}$ & 1 \\
\hline GO:0033592 & $\begin{array}{l}\text { RNA strand annealing } \\
\text { activity }\end{array}$ & 1 \\
\hline GO:0000403 & Y-form DNA binding & 1 \\
\hline
\end{tabular}

GO, gene ontology; ATP, adenosine triphosphate; JUN kinase, c-Jun N-terminal kinase; snRNA, small nuclear RNA; UTR, untranslated region.

that HELQ mainly participated in the process of DNA repair (Table III).

\section{Discussion}

DNA helicases have crucial roles in maintaining genome stability and stable DNA replication in all organisms. They have 
Table III. Biological process ontology.

\begin{tabular}{|c|c|c|}
\hline GO ID & GO term & Reliability, \% \\
\hline GO:0006281 & DNA repair & 28 \\
\hline GO:0006310 & DNA recombination & 9 \\
\hline GO:0006397 & mRNA processing & 9 \\
\hline GO:0006364 & rRNA processing & 9 \\
\hline GO:0009615 & Response to virus & 8 \\
\hline GO:0045449 & Regulation of transcription & 8 \\
\hline GO:0006314 & Intron homing & 8 \\
\hline GO:0006268 & DNA unwinding involved in replication & 8 \\
\hline GO:0000398 & Nuclear mRNA splicing, via spliceosome & 8 \\
\hline GO:0008380 & RNA splicing & 8 \\
\hline GO:0040007 & Growth & 8 \\
\hline GO:0006260 & DNA replication & 8 \\
\hline GO:0007126 & Meiosis & 8 \\
\hline GO:0006350 & Transcription & 8 \\
\hline GO:0009792 & Embryonic development ending in birth or egg hatching & 8 \\
\hline GO:0006417 & Regulation of translation & 8 \\
\hline GO:0000393 & Spliceosomal conformational changes to generate catalytic conformation & 8 \\
\hline
\end{tabular}

GO, gene ontology.

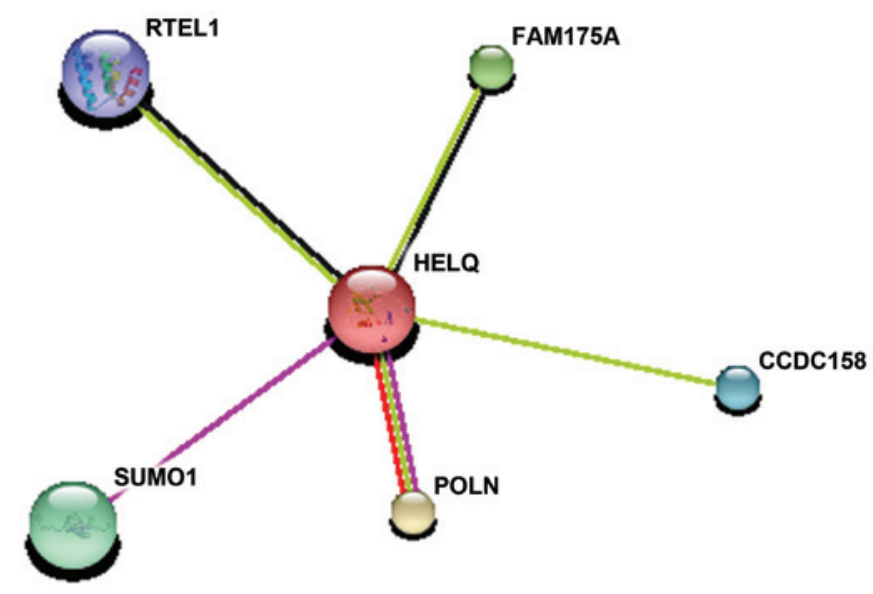

Figure 5. Cross-linked analysis of HELQ protein. RTEL1, regulator of telomere elongation helicase 1; FAM175A, family with sequence similarity 175 member A; HELQ, helicase, POLQ-like; CCDC158, coiled-coil domain containing 158; SUMO1, small ubiquitin-like modifier 1; POLN, DNA polymerase $v$.

been implicated in nucleotide excision repair, mismatch repair, base excision repair, double strand break repair and cross-link repair (14). The helicases PriA, RecG, RuvAB, RecBCD, UurD, Srs2, Rep and RecQ are well known for roles in promoting DNA repair and recombination by several possible mechanisms (15-17). Similar to RecQ, the Mus308 family of helicases supports genome stability. The Mus308 locus was identified in Drosophila melanogaster, being required for resistance to DNA crosslinking agents (4). Moldovan et al (18) found that the deletion of HEL308 in human cells could induce sensitivity to replication-blocking lesions, namely the ICLs induced by mitomycin $\mathrm{C}$ and irinotecan hydrochloride. Biochemical studies have established that human HEL308 is an ATP-dependent enzyme that unwinds DNA with a 3 '-5' polarity $(3,19)$, which is consistent with the structure of HELQ containing ATP-binding sites found in the present study. In the functional domains of HELQ, Helicase_ATP-Bind_1 and Helicase_Cter locate in the center position and they mainly perform helicase activity. At the C-terminal, the helix-hairpin-helix configuration may be important for binding to DNA strands. The results of the functional protein association networks found that HELQ was involved in ubiquitination in the process of DNA repair. RTEL1 is responsible for the maintenance of chromosome ends and has a synergistic effect with proliferating cell nuclear antigen (PCNA) in the process of DNA replication to ensure cell growth and division, and to avoid genetic mistakes. When RTEL1 cannot combine with PCNA, DNA replication cannot continue and the errors produced lead to cancer (20). FAM175A mediates the formation of the BRCA1-RAP80 complex, which adds or modifies existing ubiquitin chains to promote DNA damage repair (21). SUMO1 is important in the control genomic integrity. $\mathrm{Hu}$ et al (22) found that SUMO1 repaired DNA lesions through an ubiquitin-like modification path. POLN works as a DNA polymerase and participates in DNA repair to make DNA replication proceed normally (18). However, the exact mechanism of HELQ remains unclear.

An increasing number of studies focus on the function exploration of HELQ. Ward et al (23) found that HELQ-1 played a role in meiotic double-strand break repair by promoting postsynaptic RAD-51 filament disassembly in Caenorhabditis elegans. Another study identified that in humans, HELQ was expressed in the ovaries, testes, heart and skeletal muscle (24). Luebben et al (25) reported that mammalian HELQ contributed to genome stability in unchallenged conditions through a mechanism distinct from the function of 
Fanconi anemia complementation group C. HELQ in humans requires additional investigation, particularly the association with cancer. The occurrence of tumors is usually closely associated with abnormal DNA replication and cell proliferation. Appropriate readjustment in the structure of HELQ to change its primary function may be used to alleviate DNA damage or promote DNA variation.

The genetic complexity of cancer has posed a challenge for devising successful therapeutic treatments. Tumor resistance to cytotoxic chemotherapy drugs and radiation, which induce DNA damage, has limited their effectiveness (26). Targeting the DNA damage response is one strategy for combating cancer. The prospect for success of chemotherapy treatment may be improved by the selective inactivation of a DNA repair pathway.

In conclusion, the structure of HELQ protein was predicted and analyzed in this study. Its unique structural characteristics will have an important role in future investigations of HELQ gene deletion, as well as the etiological analysis and targeted therapy of ovarian cancer.

\section{References}

1. Thomson CA, E Crane T, Wertheim BC, Neuhouser ML, Li W, Snetselaar LG, Basen-Engquist KM, Zhou Y and Irwin ML: Diet quality and survival after ovarian cancer: Results from the Women's health initiative. J Natl Cancer Inst 106: dju314, 2014.

2. Adelman CA, Lolo RL, Birkbak NJ, Murina O, Matsuzaki K, Horejsi Z, Parmar K, Borel V, Skehel JM, Stamp G, et al: HELQ promotes RAD51 paralogue-dependent repair to avert germ cell loss and tumorigenesis. Nature 502: 381-384, 2013.

3. Marini F and Wood RD: A human DNA helicase homologous to the DNA cross-link sensitivity protein Mus308. J Biol Chem 277: 8716-8723, 2002.

4. Boyd JB, Sakaguchi K and Harris PV: mus308 mutants of Drosophila exhibit hypersensitivity to DNA cross-linking agents and are defective in a deoxyribonuclease. Genetics 125: 813-819, 1990.

5. Leonhardt EA, Henderson DS, Rinehart JE and Boyd JB: Characterization of the mus308 gene in Drosophila melanogaster. Genetics 133: 87-96, 1993.

6. Dolgova EV, Alyamkina EA, Efremov YR, Nikolin VP, Popova NA, Tyrinova TV, Kozel AV, Minkevich AM, Andrushkevich OM, Zavyalov EL, et al: Identification of cancer stem cells and a strategy for their elimination. Cancer Biol Ther 15: 1378-1394, 2014.

7. Roy S: Maintenance of genome stability in plants: Repairing DNA double strand breaks and chromatin structure stability. Front Plant Sci 5: 487, 2014.

8. Li WQ, Hu N, Hyland PL, Gao Y, Wang ZM, Yu K, Su H, Wang CY, Wang LM, Chanock SJ, et al: Genetic variants in DNA repair pathway genes and risk of esophageal squamous cell carcinoma and gastric adenocarcinoma in a Chinese population. Carcinogenesis 34: 1536-1542, 2013.
9. Gao Y, He Y, Xu J, Xu L, Du J, Zhu C, Gu H, Ma H, Hu Z, Jin $\mathrm{G}$, et al: Genetic variants at $4 \mathrm{q} 21,4 \mathrm{q} 23$ and $12 \mathrm{q} 24$ are associated with esophageal squamous cell carcinoma risk in a Chinese population. Human Genet 132: 649-656, 2013.

10. Liang C, Marsit CJ, Houseman EA, Butler R, Nelson HH, McClean MD and Kelsey KT: Gene-environment interactions of novel variants associated with head and neck cancer. Head Neck 34: 1111-1118, 2012

11. McKay JD, Truong T, Gaborieau V, Chabrier A, Chuang SC, Byrnes G, Zaridze D, Shangina O, Szeszenia-Dabrowska N, Lissowska J, et al: A genome-wide association study of upper aerodigestive tract cancers conducted within the INHANCE consortium. PLoS Genet 7: e1001333, 2011.

12. Käll L, Krogh A and Sonnhammer EL: A combined transmembrane topology and signal peptide prediction method. J Mol Biol 338: 1027-1036, 2004.

13. Scott L, Chahine J and Ruggiero J: Prediction of Protein Structures using a Hopfield Network: In: Sixth Brazilian Symposium on Neural Networks 2000. IEEE Computer Society Press, Washington, DC: pp284-284, 2000.

14. McGlynn P: Helicases at the replication fork. Adv Exp Med Biol 767: 97-121, 2013.

15. Tsang E and Carr AM: Replication fork arrest, recombination and the maintenance of ribosomal DNA stability. DNA repair (Amst) 7: 1613-1623, 2008.

16. McGlynn P and Lloyd RG: Recombinational repair and restart of damaged replication forks. Nat Rev Mol Cell Biol 3: 859-870, 2002.

17. Singleton MR and Wigley DB: Modularity and specialization in superfamily 1 and 2 helicases. J Bacteriol 184: 1819-1826, 2002.

18. Moldovan GL, Madhavan MV, Mirchandani KD, McCaffrey RM, Vinciguerra $\mathrm{P}$ and D'Andrea AD: DNA polymerase POLN participates in cross-link repair and homologous recombination. Mol Cell Biol 30: 1088-1096, 2010.

19. Tafel AA, Wu L and McHugh PJ: Human HEL308 localizes to damaged replication forks and unwinds lagging strand structures. J Biol Chem 286: 15832-15840, 2011.

20. Vannier JB, Sandhu S, Petalcorin MI, Wu X, Nabi Z, Ding H and Boulton SJ: RTEL1 is a replisome-associated helicase that promotes telomere and genome-wide replication. Science 342: 239-242, 2013.

21. Coleman KA and Greenberg RA: The BRCA1-RAP80 complex regulates DNA repair mechanism utilization by restricting end resection. J Biol Chem 286: 13669-13680, 2011.

22. Hu Y and Parvin JD: Small ubiquitin-like modifier (SUMO) isoforms and conjugation-independent function in DNA double-strand break repair pathways. J Biol Chem 289: 21289-21295, 2014.

23. Ward JD, Muzzini DM, Petalcorin MI, Martinez-Perez E, Martin JS, Plevani P, Cassata G, Marini F and Boulton SJ: Overlapping mechanisms promote postsynaptic RAD-51 filament disassembly during meiotic double-strand break repair. Mol Cell 37: 259-272, 2010.

24. Marini F, Kim N, Schuffert A and Wood RD: POLN, a nuclear PolA family DNA polymerase homologous to the DNA cross-link sensitivity protein Mus308. J Biol Chem 278: 32014-32019, 2003.

25. Luebben SW, Kawabata T, Akre MK, Lee WL, Johnson CS, O'Sullivan MG and Shima N: Helq acts in parallel to Fancc to suppress replication-associated genome instability. Nucleic Acids Res 41: 10283-10297, 2013.

26. Kelley MR and Fishel ML: DNA repair proteins as molecular targets for cancer therapeutics. Anticancer Agents Med Chem 8: 417-425, 2008. 